\title{
ESTUDO DO EQUILÍBRIO CORPORAL POSTURAL ATRAVÉS DO SISTEMA DE BAROPODOMETRIA ELETRÔNICA
}

Dra. Antonia Dalla Pria Bankoff

Ms. Paula Ciol

Dndo. Carlos Aparecido Zamai

Dndo. Ademir Schmidt

Ms. Daniela Dias Barros

Laboratório de Avaliação Postural e Eletromiografia

Faculdade de Educação Física/ UNICAMP

\section{Resumo}

O equilíbrio corporal postural além de toda sua complexidade, também está fundamentado nas relações provenientes das vias aferentes vestibulares e auditivas através do nervo craniano vestíbulo-coclear (VIII par), relacionado respectivamente com o equilíbrio e a audição, dois fatores fundamentais para se trabalhar nas questões posturais. O trabalho teve como objetivo verificar o equilíbrio corporal postural em cinco sujeitos do sexo masculino, idade compreendida entre 18 e 25 anos, com média de idade 21,2 anos. Foram analisada através da baropodometria a postura corporal estática e dinâmica medindo a superfície plantar/cm2; a distribuição do percentual (\%) de carga corporal através da região plantar, e $\mathrm{Kg} / \mathrm{cm}^{2}$. Foi estudada também, a postura corporal monopodálica direita e esquerda, com olhos abertos e olhos fechados, por um período de 03 segundos, verificando assim, o comportamento oscilatório do equilíbrio postural corporal. Quanto aos resultados referentes à superfície plantar $/ \mathrm{cm}^{2}$; distribuição do percentual (\%) de carga corporal; e $\mathrm{Kg} / \mathrm{cm}^{2}$, os resultados foram maiores tanto na parte anterior quanto na parte posterior da região plantar na análise dinâmica.Quanto as variáveis estudadas nas análises monopodálicas, os resultados demonstraram menor equilíbrio corporal com olhos fechados. No que se refere ao tempo de realização das análises referentes ao equilíbrio corporal, dois dos sujeitos conseguiu permanecer durante três segundos sobre o pé direito com os olhos abertos, e um sujeito sobre o pé esquerdo também com os olhos abertos. Com os olhos fechados, nenhum dos sujeitos envolvidos no estudo conseguiu terminar o teste.

Palavras Chaves: Postura corporal; Equilíbrio; Baropodometria; Distribuição de carga.

\section{Introdução}

Quando estudamos postura corporal automaticamente estamos estudando o sistema de equilíbrio corporal postural, porque entendemos existir uma relação de dependência entre ambos. Os reflexos de endireitamentos utilizados para a manutenção da postura se constituem importantes para que possamos entender a complexidade da postura corporal tendo em vista ser um trabalho integrado e simultâneo na postura 
corporal, e se em algumas situações esta integração for interrompida, conseqüentemente algo acontecerá, como por exemplo: Quando perdemos a seqüência lógica de passos numa caminhada, ou então quando erramos os passos numa dança em relação ao ritmo; quando perdemos o equilíbrio e tropeçamos e outros fatores, tudo isso se constitui pela interrupção momentânea no circuito integrado destes reflexos posturais. Estes reflexos de endireitamentos labirínticos, os que atuam sobre a cabeça, pescoço, corpo e os ópticos, estão situados na parte ventral do mesencéfalo, situado em frente ao terceiro par de nervos cranianos. Pouco se sabe a respeito do meio pelo qual esses reflexos contribuem para proporcionar uma postura corporal ereta e de equilíbrio, porém, sabemos o quanto eles são importantes (BANKOFF et al. 1992; DI GRAZIA, 2003; BEKEDORF, 2003; DI GRAZIA; BANKOFF, 2005).

A postura corporal envolve conceito de equilíbrio, coordenação neuro-muscular e adaptação que representa um determinado movimento corporal, e as respostas posturais automáticas são dependentes do contexto, ou seja, elas são ajustadas para ir de encontro às necessidades de interação entre os sistemas de organização postural (equilíbrio, neuro-muscular e adaptação) e o meio ambiente (BANKOFF, 1996). Muitas observações sugerem que o controle da postura não está simplesmente baseado em um conjunto de respostas reflexas, nem é uma resposta pré-programada acionada por um desequilíbrio, ao invés disto, o controle da postura é uma característica adaptável ao sistema motor, que se baseia na interação entre o estímulo aferente e a resposta eferente (ENOKA, 1995).

Na posição ereta, $25 \%$ do peso do corpo são distribuídos para cada calcâneo e $25 \%$ para a cabeça dos cinco metatarsos de cada pé; na proporção de cerca de uma parte para o I metatarso e 2,5 partes para os metatarsos II a V. A maior parte da tensão no arco longitudinal é suportada pelos ligamentos plantares. Somente cerca de 15 a $20 \%$ da tensão são suportadas pelos músculos tibiais posterior e fibular. Quando o corpo está na ponta de um pé, a tensão no arco é aumentada quatro vezes (GEHLSEN; SEGER, 1980).

O equilíbrio corporal postural, também, está fundamentado nas relações provenientes das vias aferentes (vias auditivas e vias vestibulares) através do VIII par de nervos craniano chamado vestíbulo-coclear, relacionado respectivamente com o 
equilíbrio e a audição, dois fatores fundamentais para se trabalhar com as questões posturais (BANKOFF et al. 1992).

A questão do equilíbrio na postura corporal tem sido estudada, mas faltam mecanismos no que se refere à avaliação postural. É comum o sujeito sofrer algum tipo de interferência modificando a postura corporal provenientes dos sistemas de equilíbrio e audição relacionados com nervo vestíbulo-coclear e ouvido interno, sendo os casos mais comuns relacionados com a labirintite e até mesmo zumbidos nos ouvidos (BANKOFF et al. 1992; DI GRAZIA, 2003; BEKEDORF, 2003; DI GRAZIA; BANKOFF, 2005).

Segundo Saad et al. (1997) relatam que estudos recentes em neurociências mostram que as mudanças ocorridas no sistema tônico-postural não dependem exclusivamente do ouvido interno, mas na maioria dos casos de receptores sensitivos internos e externos, sendo os mais importantes os dos olhos e os dos pés. Ainda relatam que a partir de uma reprogramação dos receptores sensitivos utilizando técnicas de manipulação corporal é possível colocá-los em ordem para restaurar o equilíbrio corporal global dos sistemas neuro-muscular, e assim, melhorar a postura corporal.

Mochizuki et al. (1998) avaliaram os parâmetros biomecânicos relacionados ao equilíbrio corporal postural em diferentes posturas, através da estabilometria, o qual serve para medir e avaliar o equilíbrio corporal postural. Estes autores mediram e avaliaram o equilíbrio corporal postural num grupo de sujeitos, em quatro horários distintos ao longo do dia com o objetivo de verificar suas relações (equilíbrio corporal postural $\mathrm{x}$ atividades físicas) com as práticas das atividades físicas. Os resultados mostraram que o equilíbrio corporal postural pode comportar-se diferentemente ao longo do dia com conseqüências bastante diferenciadas para a realização das práticas das atividades físicas, que dependem do equilíbrio corporal postural como fator fundamental para o rendimento.

Johnston et al. (1998) estudaram o efeito da fadiga sobre a performance do controle motor nos membros inferiores, utilizando um instrumento para avaliar o equilíbrio corporal postural, na postura estática unilateralmente e bilateralmente, antes e depois da prática de exercícios físicos. Os resultados mostraram haver diferença significativa no equilíbrio postural após a prática do exercício físico, diminuindo assim 
a performance do controle motor, interferindo diretamente na manutenção do equilíbrio corporal postural.

Pensando nestas questões, nossa proposta de estudo foi verificar através do sistema de Baropodometria Eletrônico, o equilíbrio corporal postural em 05 sujeitos, e estudar as variáveis: superfície por $\mathrm{cm}^{2}$, porcentagem (\%) de carga e $\mathrm{Kg} / \mathrm{cm}^{2}$ ocupada pela região plantar e equilíbrio corporal.

\section{Material e Método}

\section{Sujeitos}

Foram analisados através do Sistema Baropodômetro Eletrônico; cinco sujeitos do sexo masculino, com idades entre 18 e 25 anos, e média entre 21,2 anos.

\section{Instrumento}

Para este estudo foi utilizado um Baropodômetro Eletrônico, composto por uma plataforma modular da Physical Support Italy, formado por sensores eletrônicos de platina, revestido de captor em cacho alveolar, que reconhecem as informações do apoio plantar conservando a locomoção natural de cada sujeito.

As aquisições das imagens são precisas, instantâneas, repetíveis e não invasivas. O Baropodômetro Eletrônico modular, documenta as análises com imagens de pontos de pressão plantar medidos e armazena todas as informações adquiridas a nível estático

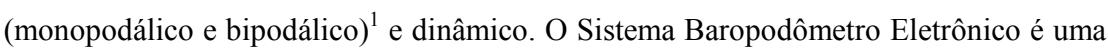
marca registrada.

\section{Aquisição de Dados}

Foram realizadas análises baropodométricas estáticas e dinâmicas, com calçado e sem calçado, e análises posturográficas monopodálicas sem calçado. Os sujeitos vestiam roupa de banho.

A análise baropodométrica estática foi realizada com o sujeito sobre a plataforma em apoio bipodálico, braços no prolongamento do corpo. A análise

\footnotetext{
${ }^{1}$ Monopodálico: Apoio em um único pé.

2 Bipodálico: Apoio em ambos os pés.
} 
baropodométrica dinâmica foi realizada com o sujeito caminhando sobre a plataforma, completando uma passada da locomoção. As análises baropodométricas (estática e dinâmica) foram realizadas com e sem calçado, com o objetivo de comparação. A análise posturográfica foi realizada com o sujeito em postura monopodálica, sobre a plataforma com olhos abertos e olhos fechados, apoiados sobre o pé esquerdo, com o joelho direito fletido. Posteriormente se realizava o mesmo procedimento apoiado sobre o pé direito, com o joelho esquerdo fletido. Esta análise foi realizada somente sem calçado. $\mathrm{O}$ aparelho foi calibrado com tempo de 03 segundos para cada procedimento realizado posturográfico.

\section{Resultados}

Os resultados referentes às médias dos valores percentuais em carga através da pressão plantar e os valores de superfície obtidos através da pressão plantar, de cinco sujeitos, utilizando-se baropodometria modular eletrônica, nas análises estática e dinâmica com calçado e sem calçado, acham-se nas Figuras de um a seis. Os resultados referentes a posturografia monopodálica olhos abertos e fechados, medindo os valores de superfície, os valores de percentuais de carga e os valores de kg, sem calçado achamse nas figuras de um a quatro.

Os resultados das análises baropodométricas (estática e dinâmica) foram tratados apenas com gráficos em forma de barras e discutidos. Para a análise posturográfica os resultados foram tratados estatisticamente através do t-test for dependent samples onde $\mathrm{p}<0,05$, o qual é aplicado em distribuições amostrais de estatísticas de pequenas amostras (WONNACOTT; WONNACOTT, 1990).

A Figura 1 mostra o percentual de distribuição de cargas corporal sobre os membros inferiores, aqui representados pela região plantar parte anterior (ossos metatarsos), nas análises baropodométricas estática e dinâmica. A média do percentual de carga dos cinco sujeitos estudados foi um pouco maior na análise dinâmica, com calçado e sem calçado. Já na análise estática, o menor valor foi registrado para o pé esquerdo, com e sem calçado. Quando se observa a Figura 2, a superfície ocupada pela região plantar parte anterior (ossos metatarsos), foi maior na análise baropodométrica dinâmica, independente das variáveis estudadas. 

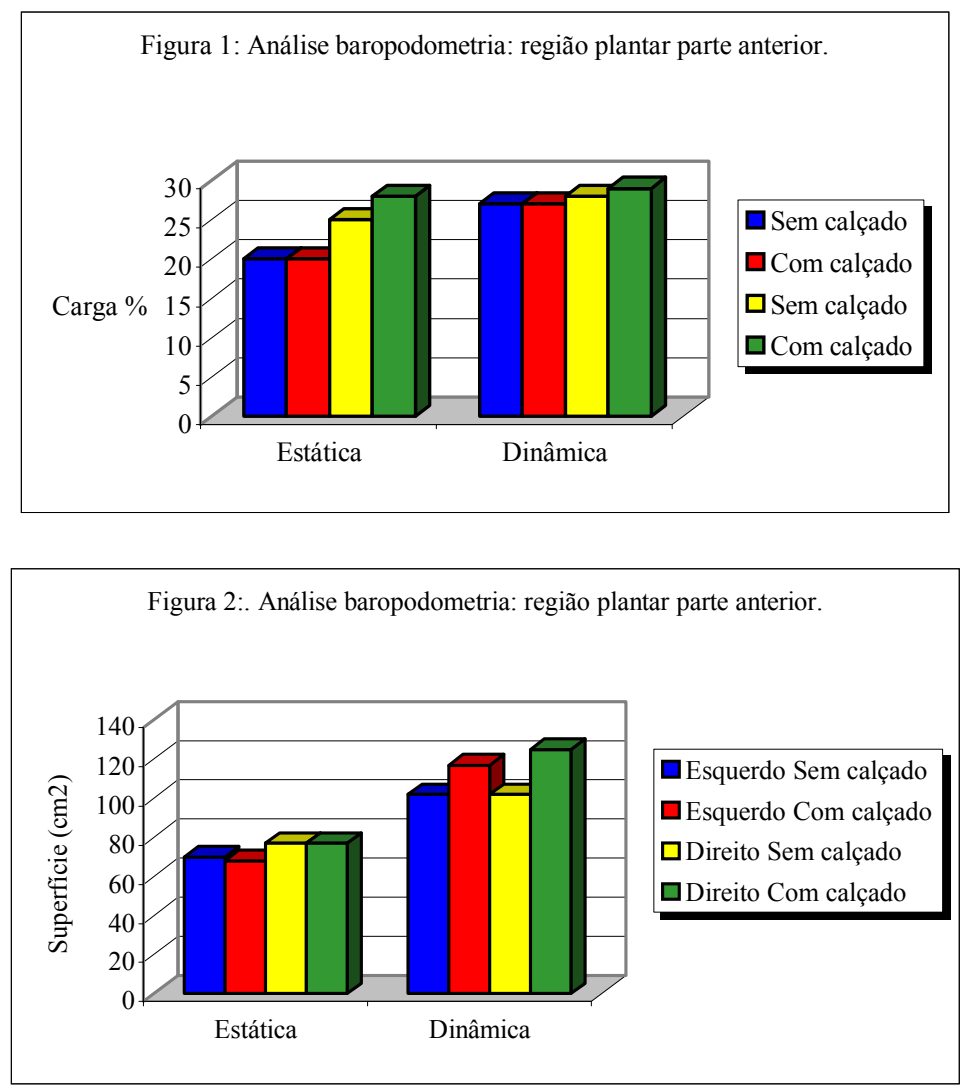

A Figura 3 representa a distribuição da superfície para a região plantar parte posterior (ossos társicos), sendo maior na análise dinâmica, especificamente pés direito e esquerdo com calçado. Quanto aos valores em percentual de cargas, mostrados na Figura 4, pela região plantar parte posterior (ossos társicos), os resultados foram maior na análise baropodométrica estática, com e sem calçado. 

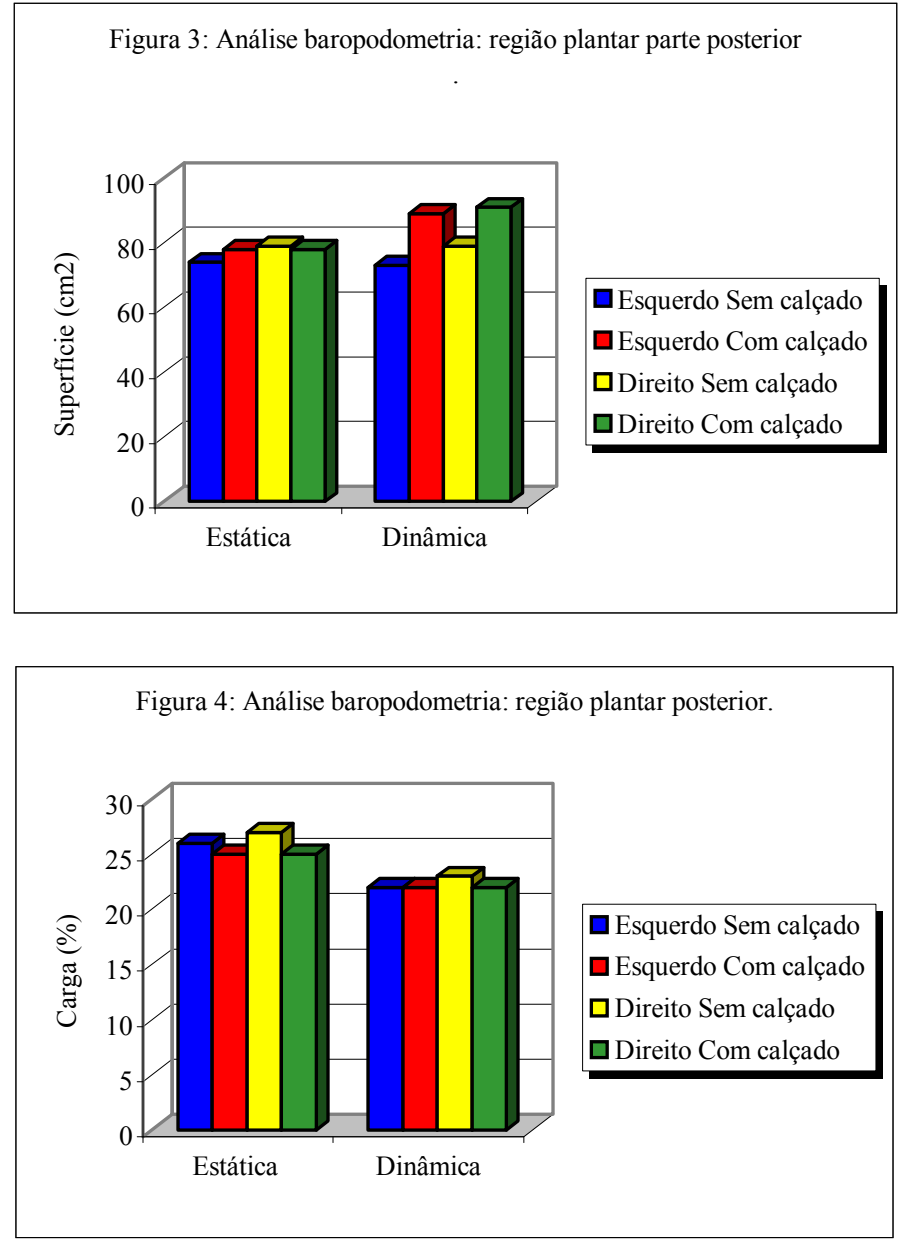


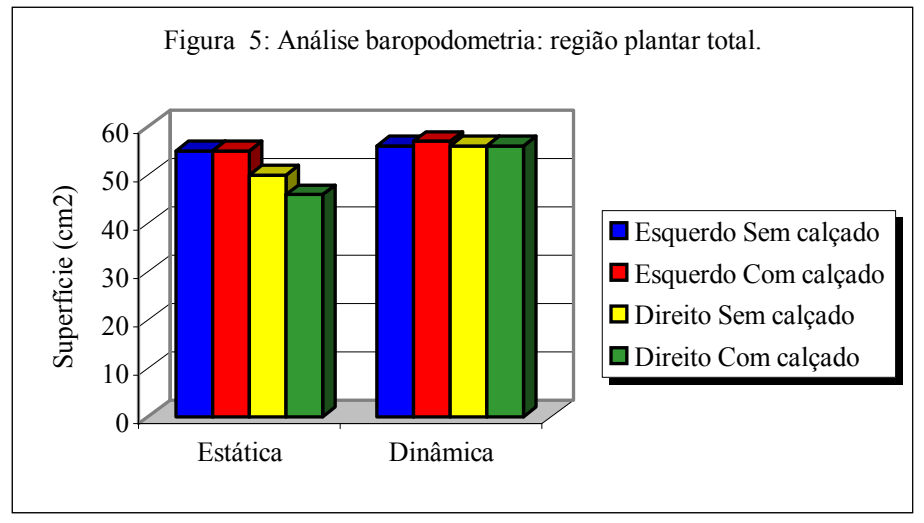

A Figura 5 mostra a distribuição da superfície da região plantar total, nas análises baropodométricas estática e dinâmica. Os resultados mostram certa uniformidade entre as análises.

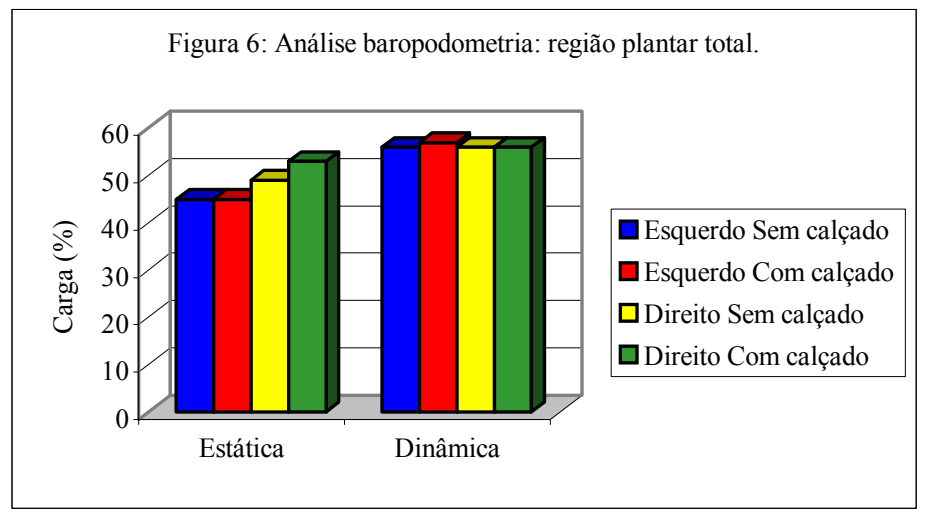

Quanto à distribuição do percentual de cargas da região plantar, o total mostrado na Figura 6, demonstra que os resultados foram maiores para postura estática, porém, com poucas diferenças para a análise dinâmica. Quanto ao estar calçado ou não, os resultados não mostraram diferenças, prevalecendo as variáveis estática e dinâmica. 
As TABELAS 1 e 2 apresentam os resultados de cada sujeito, média e desviopadrão referente à análise posturográfica monopodálicas - pés esquerdo e direito, olhos abertos e fechados, contendo 12 variáveis.

TABELA 1 - DISTRIBUIÇÃO DOS RESULTADOS REFERENTE À ANÁLISE POSTUROGRÁFICA MONOPODÁLICA PÉ ESQUERDO

\begin{tabular}{l|c|c|c|c|c|c}
\hline SUJEITOS & $\begin{array}{c}\text { 1PMOAPE } \\
\mathrm{CM}^{2}\end{array}$ & $\begin{array}{c}\text { 2PMOAPE } \\
\%\end{array}$ & $\begin{array}{c}\text { 3PMOAPE } \\
\mathrm{KG} / \mathrm{CM}^{2}\end{array}$ & $\begin{array}{c}\text { 4PMOFPE } \\
\mathrm{CM}^{2}\end{array}$ & $\begin{array}{c}\text { 5PMOFPE } \\
\%\end{array}$ & $\begin{array}{c}\text { 6PMOFPE } \\
\mathrm{KG} / \mathrm{CM}^{2}\end{array}$ \\
\hline Sujeito 1 & 162 & 49 & 34 & 145 & 48 & 40 \\
Sujeito 2 & 114 & 60 & 33 & 80 & 40 & 49 \\
Sujeito 3 & 152 & 53 & 40 & 145 & 49 & 45 \\
Sujeito 4 & 84 & 57 & 42 & 72 & 98 & 62 \\
Sujeito 5 & 162 & 82 & 59 & 170 & 98 & 62 \\
Média & 134,8 & 60,2 & 41,6 & 122,4 & 66,6 & 51,6 \\
Desvio & & & & & & \\
Padrão & 34,6 & 12,9 & 10,5 & 43,7 & 28,9 & 10,0 \\
\hline
\end{tabular}

PMOAPE: Posturografia monopodálica olho aberto pé esquerdo

PMOFPE: Posturografia monopodálica olho fechado pé esquerdo

TABELA 2 - DISTRIBUIÇÃO DOS RESULTADOS REFERENTE À ANÁLISE POSTUROGRÁFICA MONOPODÁLICA PÉ DIREITO

\begin{tabular}{l|c|c|c|c|c|c}
\hline $\begin{array}{c}\text { SUJEITO } \\
\text { S }\end{array}$ & $\begin{array}{c}\text { 7PMOAP } \\
\mathrm{D} \\
\mathrm{CM}^{2}\end{array}$ & $\begin{array}{c}\text { 8PMOAPD } \\
\%\end{array}$ & $\begin{array}{c}\text { 9PMOAPD } \\
\mathrm{KG} / \mathrm{CM}^{2}\end{array}$ & $\begin{array}{c}\text { 10PMOFP } \\
\mathrm{D} \\
\mathrm{CM}^{2}\end{array}$ & $\begin{array}{c}\text { 11PMOFP } \\
\mathrm{D} \\
\%\end{array}$ & $\begin{array}{c}\text { 12PMOFP } \\
\mathrm{D}\end{array}$ \\
\hline Sujeito 1 & 167 & 51 & 34 & 134 & 49 & 34 \\
Sujeito 2 & 181 & 64 & 38 & 128 & 36 & 37 \\
Sujeito 3 & 148 & 47 & 34 & 155 & 50 & 40 \\
Sujeito 4 & 143 & 100 & 72 & 104 & 100 & 72 \\
Sujeito 5 & 139 & 100 & 73 & 186 & 94 & 69 \\
Média & 155,6 & 72,4 & 50,2 & 141,4 & 65,8 & 50,4 \\
Desvio & & & & & & 29,1 \\
Padrão & 17,8 & 26,0 & 20,4 & 30,9 & 29,5 \\
\hline
\end{tabular}

PMOAPD: Posturografia monopodálica olho aberto pé direito

PMOFPD: Posturografia monopodálica olho fechado pé direito

Os resultados que serão apresentados a seguir (Gráficos 01 e 02), representam a comparação das variáveis entre pés direito e esquerdo, e os gráficos três e quatro 
representam a comparação das variáveis olhos abertos e fechados, verificando assim a manutenção do equilíbrio corporal postural.

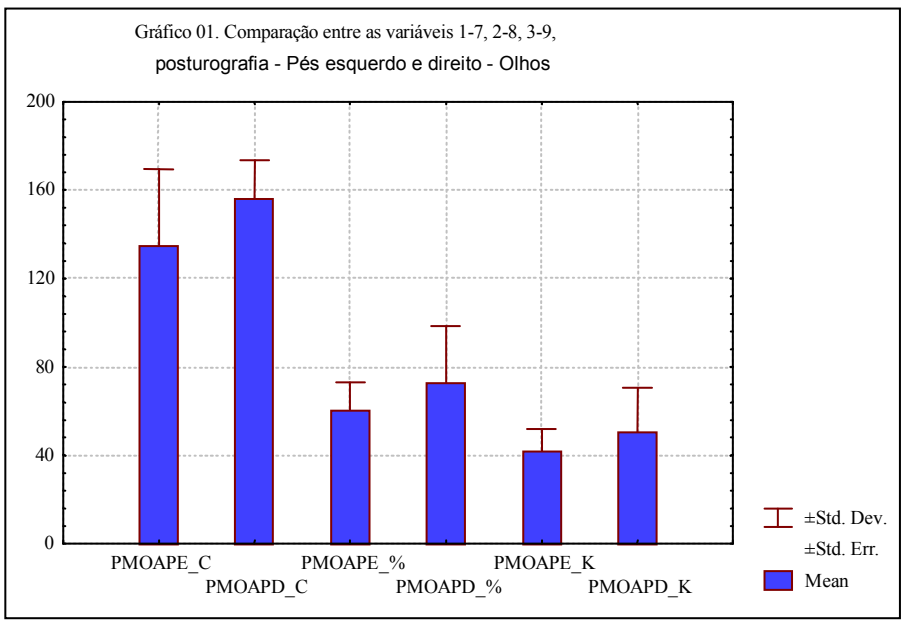

Se observarmos a Gráfico 01 que compara as variáveis estudadas e se existe diferença entre o pé esquerdo e o pé direito monopodálico, olhos abertos, verificaremos que não há diferença significativa em nível de $\mathrm{p}<0,05$ entre as variáveis estudadas. $\mathrm{O}$ Gráfico 02 compara as variáveis estudadas, porém refere-se ao pé, esquerdo e direito com os olhos fechados, verificamos não haver diferença significativa em nível de $\mathrm{p}<$ 0,05 . 

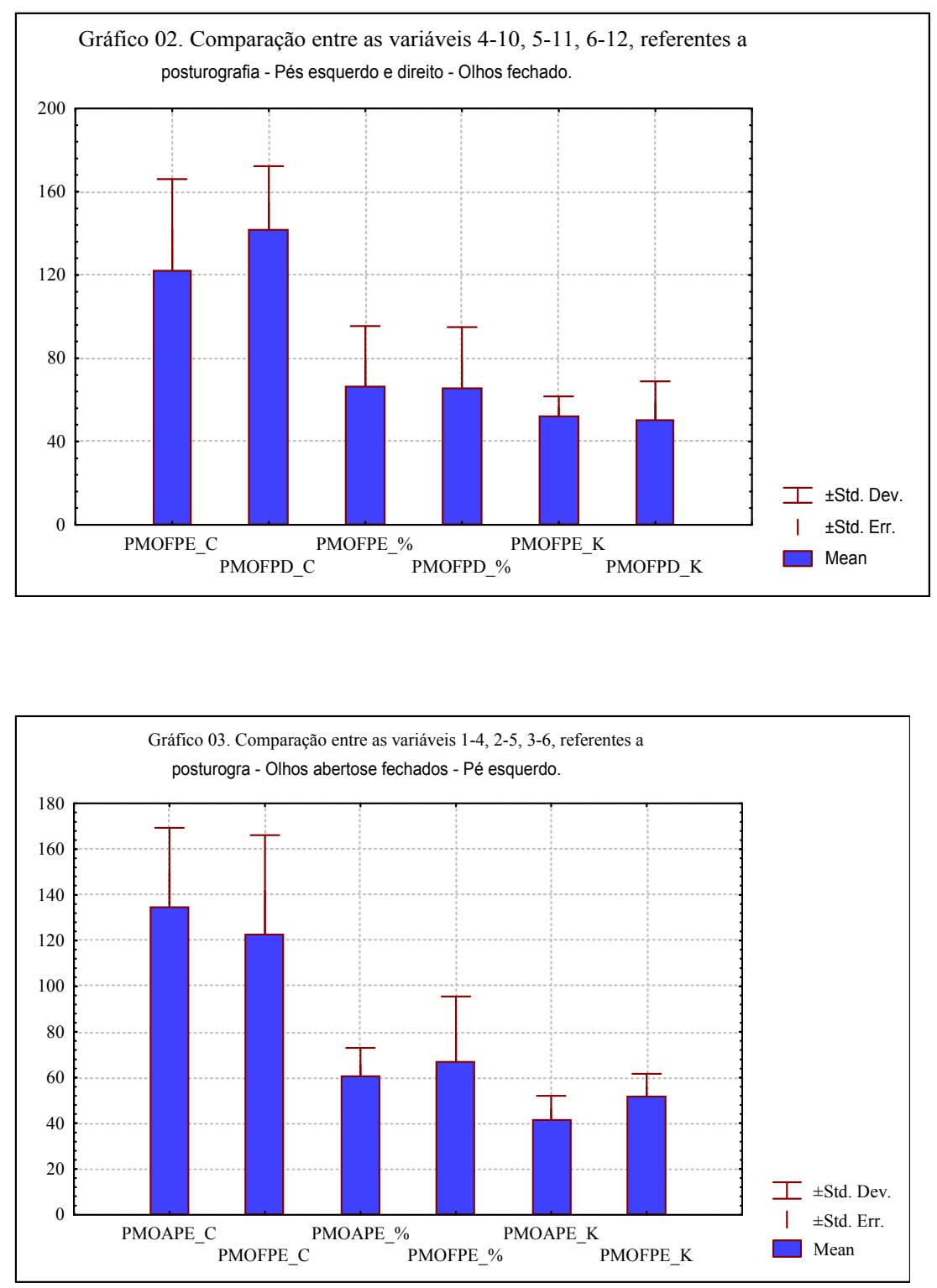
Porém, quando comparamos a relação posturográfica existente entre olhos abertos e olhos fechados, para o pé esquerdo (Gráfico 03), apenas a variável $\mathrm{Kg} / \mathrm{cm}^{2}$ mostrou ser significativo em nível de $p<0,05$, as demais não mostraram significância. O Gráfico 04 compara as variáveis estudadas, olhos abertos e olhos fechados, pé direito, não mostrou significância em nível de $\mathrm{p}<0,05$.

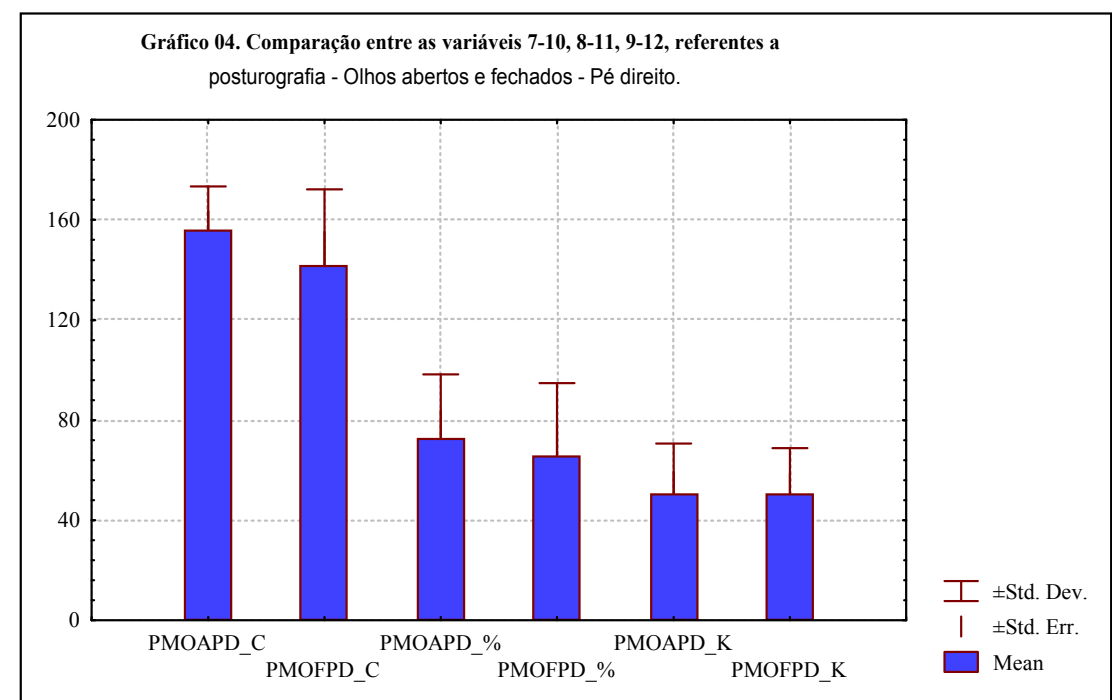

esquerad oinos abertos um aos sujeitos estudaao conseguiu permanecer ate ao termino dos 03 segundos. Na postura monopodálica direita olhos fechados apenas um dos sujeitos estudado conseguiu permanecer até 1,5 segundos; dois até 2,4 segundos, os demais permaneceram apenas 1,3 segundos. Na postura monopodálica olhos fechados a perna esquerda, um sujeito conseguiu permanecer 1,3 segundos, os demais conseguiram efetuar o teste com tempo de 2,3 segundos.

\section{Discussão}

A postura corporal é uma resposta neuromecânica que se relaciona com a manutenção do equilíbrio, e um sistema está em equilíbrio mecânico quando o 
somatório das forças que agem sobre o sistema é igual zero $\left(\sum \mathrm{F}=0\right)$. Entretanto, este sistema tem estabilidade somente se após uma perturbação ele retorna a sua posição de equilíbrio, lembrando que quando se fica numa posição em pé ereta, o corpo oscila para frente e para trás. A atividade muscular que evita que se perca o equilíbrio e se caia, representa a atividade de controle automático da postura (ENOKA, 2000).

O equilíbrio corporal dinâmico difere do equilíbrio estável, no qual a situação se modifica constantemente, e existem relativamente poucas posições momentâneas ou nenhuma, em que se cumpram as condições do equilíbrio estável (BANKOFF et al. 1992). Ainda esta autora relata que as posturas corporais diárias se registram num curto período, ou seja, não mais do que 03 segundos para sofrer qualquer tipo de alteração momentânea. As mudanças observadas no percentual de distribuição de cargas entre as análises baropodométricas estática e dinâmica, mostram a relação existente entre o equilíbrio e as posições posturais, ou seja, a manutenção do equilíbrio corporal postural se modifica numa velocidade de milésimos de segundo. Os valores mostrados na Figura 1, quanto à distribuição de percentual de cargas e a superfície (Figura 2) ocupada pela região plantar parte anterior, foram maiores na dinâmica, com e sem calçado. Os autores Hamil e Knutzen (1999), Rasch e Burke (1977), descrevem que as cargas são maiores, 1,6 em condições de marcha e 3,2 na corrida quando comparadas com a postura estática. A relação estar ou não calçado não marcou diferença, porém, a postura corporal é que registrou diferença (estática x dinâmica).

Quando observamos a Figura 3 e 4 quanto à superfície e a distribuição de percentual de cargas ocupada pela região plantar parte posterior com e sem calçado, observamos uma certa similaridade entre os resultados quando comparadas com as posturas baropodométricas estática e dinâmica.

Porém, em relação ao resultado registrado pela região plantar total, houve uma pequena diferença em relação ao percentual de cargas entre as análises baropodométricas estática e dinâmica, sendo maior para a baropodometria dinânica, confirmando mais uma vez ser a sobrecarga do peso corporal maior na postura dinâmica. Tribastone (2001), quanto à posição ereta dinâmica, relata que todo o peso do corpo recebido pelo astrágalo é transmitido: anteriormente, sobre os metatarsos, se o apoio no terreno acontece sobre a parte anterior do pé; posteriormente, sobre o 
calcâneo, se o apoio no terreno acontece sobre o calcanhar; e 57\% sobre o calcâneo e $43 \%$ sobre a parte anterior do pé, se o apoio for sobre toda a planta do pé. Ainda o mesmo autor relata que quase todos os autores estão de acordo em estabelecer que o apoio na parte posterior do pé acontece sobre a tuberosidade do calcâneo, e para a parte anterior é sobre a crista do terceiro metatarso.

Bankoff et al. (1992), relata que as relações reflexas de sensibilidade, com a velocidade do olho durante os movimentos de condução das passadas na locomoção humana, estão diretamente ligadas com a manutenção da postura corporal.

Os Gráficos 01, 02, 03, 04, mostram as comparações das variáveis estudadas, através de análises posturográficas monopodálicas, olhos abertos e fechados pés direito e esquerdo sem calçado. Convém verificar que somente a variável $\mathrm{Kg}$ pé esquerdo olhos abertos/olhos fechados foi significativa, as demais não foram significativas a nível de $p<0,05$. Embora tenham sido registradas diferenças entre as variáveis estudadas para os sujeitos, quando analisadas estatisticamente a maioria delas não foram significativas quando comparadas com olhos abertos e olhos fechados. (DI GRAZIA, 2003; BEKEDORF, 2003; DI GRAZIA; BANKOFF, 2005).

Convém ressaltar também a importância do sistema visual para a manutenção do equilíbrio corporal postural, embora os resultados não tenham sido significativos quando comparados com olhos abertos e fechados, e também lembrar, que a maioria dos autores, especificamente os estudiosos das áreas de neuro-anatomia, neurofisiologia e neuro-biomecânica referem-se muito claramente em seus estudos sobre equilíbrio e manutenção do equilíbrio corporal postural, à importância dos sistemas proprioceptivos e o meio ambiente para a manutenção do equilíbrio corporal postural. Também relatam, que a chamada "postura estática" tem mostrado ser impossível, pois sempre existe uma oscilação, que traduz uma situação dinâmica com indispensáveis e contínuos ajustes da posição destinados a manter o equilíbrio.

Tribastone (2001) relata a importância da superação de limites em treinamento quando fechamos nossos olhos normalmente ou com eles vendados, isso porque o ser humano se julga normal, ou seja, quando ele possui um sistema de visão normal, ele confia quase que $100 \%$ no seu sistema de equilíbrio e manutenção de equilíbrio corporal postural, sobre os olhos ou sobre o sistema de visão, utilizando muito pouco 
do sistema vestíbulo coclear. Para isso, ele sugere realizarmos qualquer tarefa com os olhos vendados para superação dos limites.

\section{Considerações Finais}

Convém lembrar que o equilíbrio e a manutenção do equilíbrio corporal postural é algo muito difícil em qualquer idade, e são necessários muitos estudos para afirmar com segurança qual a melhor e mais eficiente postura corporal. Alguns fatores são importantes para melhorar o equilíbrio e a manutenção do equilíbrio corporal postural, como por exemplo:

- praticar atividades físicas diariamente;

- realizar exercícios de alongamento;

- criar o hábito de praticar exercícios de relaxamento;

- praticar exercícios respiratórios diariamente;

- criar o hábito de sessões de massoterapia;

- realizar pequenos percursos caminhando com olhos vendados, iniciando primeiro com um olho aberto e outro fechado, e depois de algum tempo treinar com ambos fechados, lembrando que este tipo de prática deve ser realizado sempre em companhia de uma pessoa, de preferência de mãos dadas;

- realizar exercícios de balançar os membros superiores e membros inferiores, lembrando que para balançar os membros inferiores é necessário estar apoiado.

Toda orientação aqui citada é importante pelo fato de alternância de ativação de diferentes cadeias musculares, lembrando que as respostas posturais entre os membros são importantes quando os dois membros estão sendo utilizados para dar apoio a uma posição. Com os resultados encontrados é possível concluir que na postura dinâmica o percentual de carga e a área de superfície são maior quando comparada com a postura estática, independente do sujeito estar com ou sem calçado, para as regiões plantar anterior, posterior e plantar total, nas análises baropodométrica. Quanto às análises posturográficas, a única variável que mostrou ser dependente entre olhos abertos e fechados foi $\mathrm{Kg} / \mathrm{cm}^{2}$ para o pé esquerdo. 


\begin{abstract}
Body posture balance is based on the relations originating from the vestibule and auditory afferent ways through the crania nerve vestibulum-cochlear (VIII pair), related respectively to a balance and hearing. These two factors are responsible for working with questions of postures. The purpose of this work was to verify the body posture balance in five male individuals (age between 18 and 25, average of age 21,2 years old). The static and dynamic body posture had been analyzed using a Baropodometer, measuring the surface by square centimeter and the percentage distribution of the body load through the plantar region. It was also studied the right and left monopodalic body posture, with open and closed eyes, for a period of tree seconds. That way it was verified the oscillatory behavior of the body posture balance. Regarding the results referring the surface by square centimeter and the percentage distribution of body load, the rear foot and forefoot plantar region, in the dynamic analysis, was bigger. Regarding the variables studied in the monopodalic analyses, the results showed smaller with closed eyes, decreasing this way the body posture balance. Concerning the time of the analyses of the body balance, two of the individuals remained for a period of tree seconds standing on the right leg with opened eyes and another individual on the left leg also with opened eyes. None of the individuals involved in the study finished the test.
\end{abstract}

Key Words: Body posture balance; Baropodometer; Load distribution.

\title{
Referências Bibliográficas
}

BANKOFF, A. D. P. Postura corporal: fatores biológicos da postura ereta: causas e conseqüências. Brasília: Ministério da Saúde: Ministério da Educação e do Desporto, 1996

. et al. Analisis poddometrico de los atletas de levantamiento de peso mediante la tecnica video-pedometrica. In: CONGRESSO CIENTÍFICO OLÍMPICO, 1992, Málaga. Anais... Málaga, 1992.p. 18

BEKEDORF, R. G. Análise do equilibrio estático através de um baropodômetro eletrônico. 2003. Monografia (Trabalho de Conclusão de Curso)-Faculdade de Educação Física, Universidade Estadual de Campinas, Campinas, 2003.

DI GRAZIA, R. C. Alterações posturais relacionadas com a disfunção da articulação temporomandibular e seu tratamento. 2003. Dissertação (Mestrado em Educação 
Física)-Faculdade de Educação Física, Universidade Estadual de Campinas, Campinas, 2003.

DI GRAZIA, R. C.; BANKOFF, A. D. P. Alterações posturais relacionadas com a disfunção da articulação temporomandibular e seu tratamento. EF Deportes: revista digital. Disponível em: < http:// www.efdeportes.com, 2005.

ENOKA, M. R. Bases neuromechanical of kinesiology. $2^{\text {nd }}$ ed. Champaing: Human Kinetics. 1995.

GEHLSEN, G. M.; SEGER, A. Selected measures of angular displacement, strength and flexibility in subjects with and without skin splints. Research Quarterly, v. 51, n. 3, p. $478-485,1980$.

HAMILL, J.; KNUTZEN, K.M. Bases do movimento humano. São Paulo: Manole, 1999.

JOHNSTON, R. B. et al. Effect of lower extremity muscular fatigue on motor control performance. Medicine and Science in Sports and Exercise, v. 30, n. 12, p. 1703-1707, 1998.

MOCHIZUKI, L. et al. Estudo biomecânico sobre o controle do equilíbrio postural em função de ritmos circadianos. Revista Brasileira de Postura e Movimento, v. 2, n. 1, p. 51-55, 1998.

RASCH, P. J.; BURKE, R. K. Cinesiologia e anatomia aplicada. 5. ed. Rio de Janeiro: Guanabara Koogan, 1977.

$\mathrm{SAAD}, \mathrm{M}$. et al. Sinais clínicos associados a prognóstico de marcha em paralisia cerebral espástica. Revista Brasileira de Postura e Movimento, v. 1, n. 1, p. 5-12, 1997. 
TRIBASTONE, F. Tratado de exercícios corretivos: aplicados à reeducação motora postural. São Paulo: Manole, 2001.

WONNACOTT, T. H.; WONNACOTT, R. J. Introductory statistics. $5^{\text {th }}$ ed. New York: J. Wiley, 1990. 